\title{
LAMINAR-TURBULENT FLOW AROUND A WEDGE PLACED ON SHARP AND BLUNTED PLATES
}

\author{
V. Borovoy, V. Mosharov, V. Radchenko, and A. Noev \\ Central Aerohydrodynamic Institute (TsAGI) \\ Zhukovsky, Moscow Region 140180, Russia
}

The flow structure and heat transfer on sharp and blunted plates near a wedge have been studied experimentally at Mach number 6 and two Reynolds numbers corresponding to laminar and transitional boundary layers ahead of the wedge. Similarly to the two-dimensional (2D) flow, the plate's blunting causes a decrease of heat transfer at threedimensional (3D) interference between the boundary layer and the shock wave. However, there is a threshold bluntness value beyond which the influence is negligible. Heat transfer and pressure measurements as well as surface flow visualization have been performed by means of panoramic (global) methods based on the use of luminescent coatings. They have been used in a short duration (pulse) wind tunnel for the first time.

\section{INTRODUCTION}

Interaction between the boundary layer and oblique shock generated by a wedge placed on the plate is the subject of many investigations (see survey [1]). Even the first studies (see, for example, [2]) showed that heat transfer increases several times behind the shock. It has been also demonstrated [2-8] that the flow at some distance from the leading edge of a sharp wedge is similar to a conical one with the pole located near the wedge's leading edge, and the flow characteristics vary mildly along the rays originating from the pole. Under these conditions, flow characteristics distributions in the cross sections orthogonal to the rays are similar to the $2 \mathrm{D}$ interference distributions. Some authors have studied this quasi-conical flow in detail.

Intensive numerical investigations of turbulent flow around wedges placed on a plate have been performed using different simulation approaches during the last two decades along with experiments [7]. Numerical simulations adequately

This is an Open Access article distributed under the terms of the Creative Commons Attribution-Noncommercial License 3.0, which permits unrestricted use, distribution, and reproduction in any noncommercial medium, provided the original work is properly cited. 
describe the main features of the real flows but the calculated values of heat flux and shear stress significantly differ from the experimental ones for the developed separation regions generated by the strong shocks. It was established [1] that fuller experimental information for wide flow classes is necessary to improve and verify the calculation codes.

Almost all previous investigations have focused on interference flows on sharp plates or cones. But a recent study of $2 \mathrm{D}$ laminar flow on a plate $[9,10]$ has demonstrated that even a small bluntness of the plate results in significant decrease of heat transfer in shock impinging region and the maximum heat flux decreases at the bluntness radius only up to a certain threshold value [11]. These features are related to the presence of a high entropy layer generated by the blunt leading edge. The present study (see also [12]) focuses on the influence of bluntness at $3 \mathrm{D}$ transitional flow around a wedge.

In most previous experiments, local measurements have been performed by discrete sensors which are not very effective at 3D flows. Global (panoramic) methods are more adequate for such flows $[8,13]$. The present study develops optical methods based on luminescent coatings. These methods are adopted in a short duration (pulse) wind tunnel to measure heat flux and pressure distribution as well as visualization of surface streamlines on the model's surface [14].

\section{MODEL AND FLOW CHARACTERISTICS}

Two similar models have been tested (Fig. 1). The first one is intended for heat transfer investigations with Temperature Sensitive Paints (TSP). The main part of the model (the plate 1 ) is made of prepreg resin and covered with white epoxy paint of 0.1-millimeter thickness. Two thermocouples are installed on the plate to measure the initial surface temperature $T_{w}$. The length of the plate $L$ is $319 \mathrm{~mm}$, its width $B=150 \mathrm{~mm}$. An additional steel plate 4 of 3 -millimeter thickness is attached to the front surface of the main plate in order to get a sharp leading edge. An adapter 5 can be installed on this plate to change the bluntness radius of the leading edge in the range from 0 to $4 \mathrm{~mm}$.

The second model is intended for pressure distribution measurements with Pressure Sensitive Paints (PSP) and surface flow visualization with luminescent particles. Its main plate 1 is made of aluminum alloy; therefore, the additional plate 4 is not necessary for this model. The same adapters 5 are used for leading edge bluntness variations. Sharp wedges of different angles $\left(\theta=10^{\circ}, 15^{\circ}\right.$, and $20^{\circ}$ ) can be attached to the main plate. The distance between the wedge's leading edge and the sharp leading edge of the plate is $X_{0}=129 \mathrm{~mm}$. Sharp fences 3 are used to prevent gas cross flow.

The tests were performed in a short duration wind tunnel UT-1 operating in Ludwieg tube mode at Mach number 6. A profiled nozzle with exit diameter 


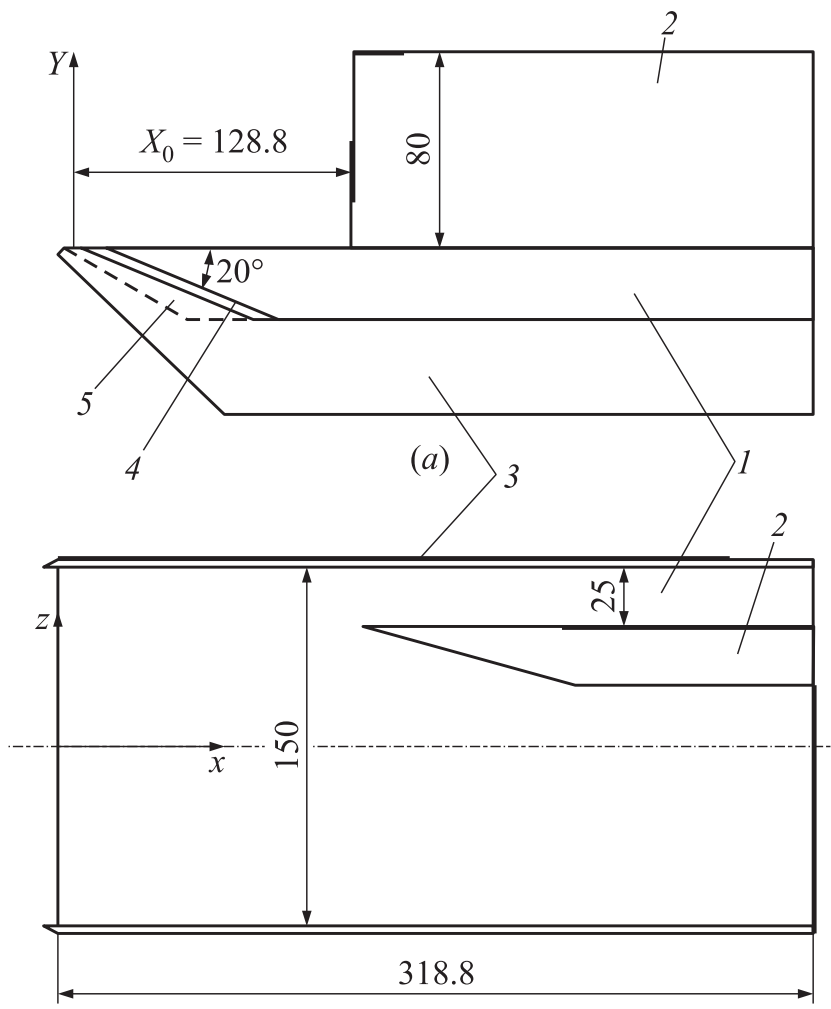

(b)

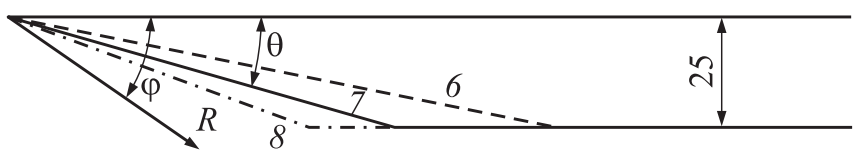

(c)

Figure 1 Model scheme: $(a)$ and $(b)$ plate with a wedge, $(c)$ wedges; 1 - main plate, 2 - wedge, 3 - fences, 4 - additional plate, 5 - adapter, $6-8$ - wedges with different angles $\theta, 6-\theta=10^{\circ}, 7-15^{\circ}$, and $8-20^{\circ}$. Dimensions are in millimeters

$300 \mathrm{~mm}$ was used. The duration of the steady flow was $40 \mathrm{~ms}$. Two test cycles were conducted. They differ mainly in total pressure and total temperature. The averaged flow parameters for both cycles are presented in Table $1\left(P_{0}\right.$ is the total pressure, $T_{0}$ is the total temperature, $T_{w}$ is the wall temperature before the test, and $\operatorname{Re}_{\infty, L}$ is the Reynolds number based on the undisturbed flow parameters and the model length $L$ ). 
Table 1 Averaged flow parameters for two test cycles

\begin{tabular}{ccccc}
\hline Test cycle & $P_{0}$, bar & $T_{0}, \mathrm{~K}$ & $T_{w}, \mathrm{~K}$ & $\mathrm{Re}_{\infty, L}$ \\
\hline 1 & 32.4 & 726 & 292 & $4.7 \cdot 10^{6}$ \\
2 & 94.8 & 578 & 296 & $19.2 \cdot 10^{6}$ \\
\hline
\end{tabular}

\section{MEASUREMENT METHODS}

\subsection{Measurement of Heat Transfer}

Heat transfer investigations in long duration wind tunnels have been performed with melting TSPs for a long time (see, for example, [4]). Luminescent paints were first used in such investigations in the 1990s in the USA [13]. In the present study, luminescent TSP was used for heat transfer investigations in a short duration (pulse) wind tunnel (see also [14, 15]).

The method is based on temperature quenching of luminescence of an organic luminophor. Heat flux is determined by temperature increase during a known time interval in each point of the model's surface.

A binary (two-color) TSP was used in the present tests. The binary paint contains two types of organic luminophor molecules. One luminophor is temperature sensitive. Its luminescence decreases with temperature growth. The other luminophor is temperature insensitive. Its luminescence is used as a reference for the pixel-by-pixel correction of excitation light intensity variation from flash to flash. Both luminophors are excited simultaneously by an ultraviolet (UV) (260-390 nm) flash lamp, but they emit light in different spectral ranges. The temperature sensitive luminescence is red and the temperature insensitive luminescence is blue; therefore, they can be separated spectrally. The intensity of the emitted red light decreases at temperature increase at the rate of $3 \%-5 \%$ per $1{ }^{\circ} \mathrm{C}$ and is absolutely insensitive to pressure.

For each wind tunnel run, two luminescent images of the model's surface (red $I^{r}$ and blue $I^{b}$ ) are acquired before the test at a known model's surface temperature (reference images $I_{R}$ ) and two images in the flow (working images $I_{W}$ ). The ratio of these four images $I_{W}^{r} I_{R}^{b} /\left(I_{R}^{\prime} I_{W}^{b}\right)$ is sensitive only to the temperature and insensitive to the thickness of the TSP layer and to the excitation light intensity if these images are registered correctly. More detailed information can be found in $[15]$.

Heat flux is calculated based on the temperature measurements using an exact solution of a one-dimensional (1D) heat-transfer equation for a semiinfinite body. The results are presented in the form of a dimensionless heat flux (Stanton number) field: 


$$
\mathrm{St}=\frac{h}{\rho_{\infty} u_{\infty} c_{p}}=\frac{q}{\left(T_{r}-T_{w}\right) \rho_{\infty} u_{\infty} c_{p}}
$$

where $\rho_{\infty}$ and $u_{\infty}$ are the density and velocity of a free stream; $c_{p}$ is the specific heat at constant pressure; and $q$ is the heat flux. Not determining the exact values of the recovery temperature $T_{r}$ in complex laminar-turbulent and separated flows, this temperature was taken equal to the stagnation temperature $T_{0}$ on the whole surface of the model.

Each model configuration at both total pressures was tested twice. A comparison of repeated runs showed good repeatability of results if variations of the total pressure and temperature are taken into account. The mean discrepancies are about $3 \%$.

\subsection{Measurement of Pressure Fields}

Pressure fields were investigated by the PSP method which is based on the phenomenon of luminescence quenching of organic luminophors by air oxygen. The luminescence quenching phenomenon was discovered in 1935 by Kautsky and Hirsch [16]. At the same time, it was proposed to use this phenomenon for oxygen traces detection. Subsequently, there were many papers regarding the development of oxygen luminescent sensor. Ardasheva et al. were the first to propose, in 1985, to use this phenomenon for contactless pressure measurements in the gases with constant oxygen concentration, e.g., for air pressure measurements [17].

The PSP method is widely used in long duration wind tunnels for subsonic and transonic flow investigations and, rarely, for supersonic flow investigations. Its application for hypersonic flows is problematic because of significant PSP temperature sensitivity. In the present work (see also $[18,19])$, the problem was resolved $(i)$ by conducting the tests in a short duration wind tunnel; $(i i)$ by manufacturing the model from a heat-conducting material (an aluminum alloy) in contrast to model for heat transfer measurements; and (iii) by applying a fast response PSP.

The response time of PSP is determined by oxygen diffusion in the polymer layer and is directly proportional to the square of polymer layer thickness. The use of a permeable polymer (polydimethylsiloxane) applied as a very thin layer (about $2 \mu \mathrm{m}$ ) allows to get response time less than $5 \mathrm{~ms}$.

The methodology of pressure field measurements is quite similar to the temperature field measurements described above, but the uni-component PSP was used for pressure measurements in the present tests. For correction of the excitation light intensity variation, a spot of Luminescent Reference Paint (LRP) was applied to the model's surface. Luminescent reference paint is a luminescent material insensitive to the temperature and pressure. Each image is divided on the average LRP intensity. 
Pressure fields are presented as isobars of nondimensional pressure (pressure coefficient $C_{p}$ ):

$$
C_{p}=\frac{P-P_{\infty}}{0.5 \rho_{\infty} u_{\infty}^{2}}
$$

where $P_{\infty}$ is the static pressure that was calculated from the total pressure.

\subsection{Surface Streamline Visualization}

Conventional methods of surface streamline visualization (minitufts and oil flow) cannot be used in the wind tunnel UT-1 with the flow duration of $40 \mathrm{~ms}$. Particle Image Surface Flow Visualization (PISFV) method suggested recently [19, 20] allows to realize such visualization. The idea of the new method is to register small shift of oil film applied to the tested surface and to restore the complete pattern of surface flow numerically. For this purpose, some optically contrast hard particles are added to the oil film and two images of particle distribution are acquired at the investigated test parameters at some time interval. These pairs of images are processed by cross-correlation analysis similar to the kind used in Particle Image Velocimetry method to obtain the parameters of particles movement. The magnitude of the particles shift is proportional to the shear stress value (if oil thickness is a constant on the tested surface) that allows to visualize the field of shear stress. The direction of the particles shift is coincident with the direction of the surface streamline that allows to restore the surface streamline pattern on the model's surface. The method was tested in a subsonic long duration wind tunnel [20] and demonstrated its advantages over the conventional oil flow visualization. Since only a small shift of oil film is analyzed, several flow regimes can be investigated during one wind tunnel run without a new model's preparation.

The described tests have been the first experience of PISFV application at hypersonic flows. In a short duration wind tunnel, the first image can be acquired before the wind tunnel's run, the second one at the end of the run.

In short duration facilities, the model oscillates after the step load action, and, as a result, the model's positions in the first and second images can be different. To compensate for the shift of the model in the images, a set of luminescent markers attached to the model's surface was used. The model shift vector as a whole was determined by the markers coordinates in the images and the oil shift vectors on the model's surface were calculated as a vector difference between the particles shifts and the model's shift.

The model's oscillation also produces inertial force that affect on the oil film. This inertial force is compatible with aerodynamic shear stress in the region of undisturbed flow on the flat plate. As a result, some dummy streamline directions take place in the undisturbed region. 


\section{INVESTIGATION OF HEAT TRANSFER AND FLOW STRUCTURE}

\subsection{A Plate Without Wedge}

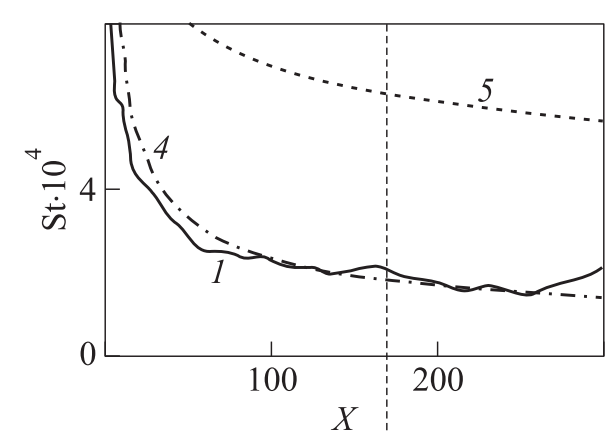

(a)

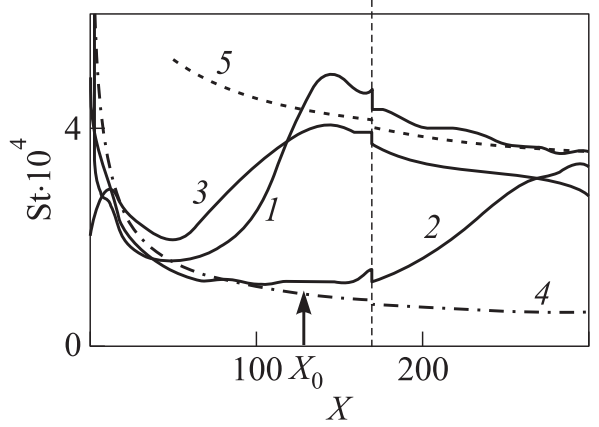

(b)

Figure 2 Stanton number distribution along the plate without wedge: (a) $\operatorname{Re}_{\infty, L}$ $=4.7 \cdot 10^{6},(b) \operatorname{Re}_{\infty, L}=19.2 \cdot 10^{6} ; 1-3-$ experiments $(1-r=0,2-1$, and $3-$ $r=2 \mathrm{~mm}), 4-5-$ calculations, $(4-$ laminar, and 5 - turbulent) $X_{0}$ - location of the wedge's leading edge in the experiments with a wedge
This investigation aimed to diagnosing the state of the boundary layer.

Figure 2 presents the distribution of Stanton number on the plate at two Reynolds numbers: $\operatorname{Re}_{\infty, L}=4.7 \cdot 10^{6}$ and $19.2 \cdot 10^{6}$. The boundary layer is laminar on almost all the length of the considered section at lower Re value (Fig. 2a).

At higher Re value (Fig. 2b), the laminar-turbulent transition on the sharp plate $(r=0)$ and on the plate with the bluntness radius $r=2 \mathrm{~mm}$ starts at the distance $X \approx 50 \mathrm{~mm}$ from the leading edge $\left(X_{*}=X / L \approx 0.16\right)$, i.e., at $\operatorname{Re}_{\infty, X} \approx 3 \cdot 10^{6}$, and ends at $X \approx 150 \mathrm{~mm}\left(X_{*} \approx 0.47\right)$, i.e., at $\operatorname{Re}_{\infty, X} \approx 9 \cdot 10^{6}$. On the plate with intermediate bluntness $r$ $=1 \mathrm{~mm}$, the transition starts at $X \approx 100 \mathrm{~mm}\left(X_{*} \approx 0.31\right)$ and does not end on the plate.

It means that the boundary layer on the plate in the region where it interferes with the wedge's shock wave is laminar at the lower Reynolds number and is transitional at the higher one.

\subsection{A Plate with 15 Degree Wedge}

Isobars and surface streamlines on the plate near the wedge with the angle $\theta$ $=15^{\circ}$ are shown in Fig. 3. Such intensive shock provokes separation not only of the laminar but also of the turbulent boundary layer (see, e.g., [8]). The boundary layer separates ahead of the shock and reattaches behind it. The 


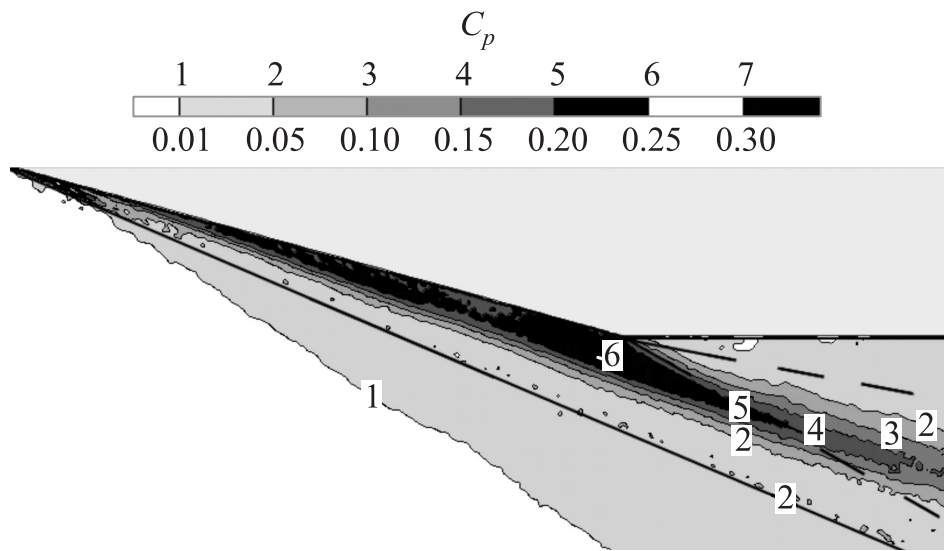

(a)

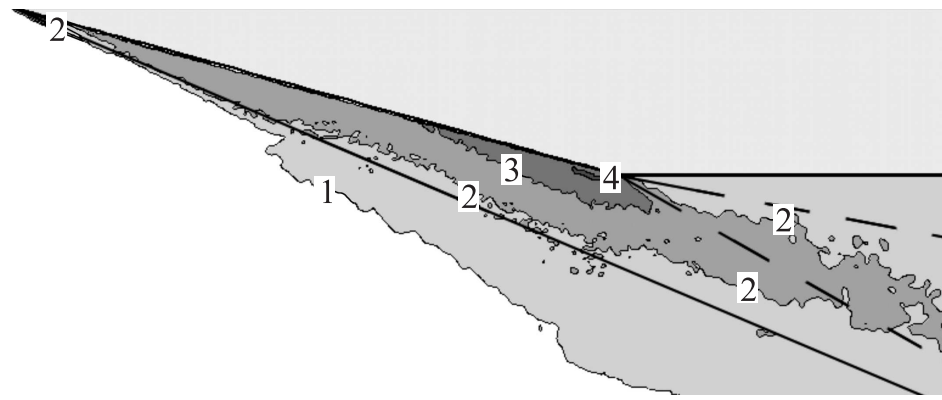

(b)

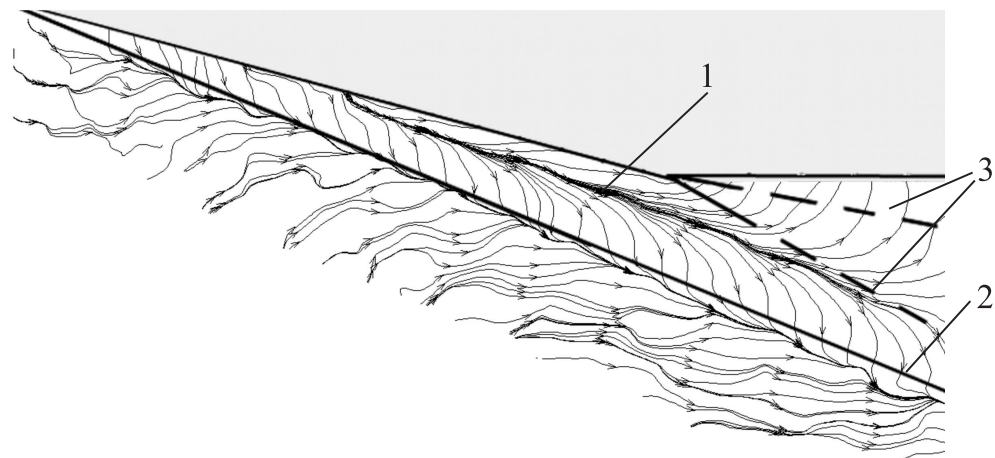

(c)

Figure 3 Isobars and surface stream lines on sharp and blunt plates in the neighborhood of wedge with $\theta=15^{\circ}$ at $\operatorname{Re}_{\infty, L}=19.2 \cdot 10^{6}$ : $(a)$ and (b) isobars; (c) surface streamlines; $(a)$ and $(c) r=0 ;(b) r=1 \mathrm{~mm} ; 1$ - diverging line, 2 - solid line shock wave, 3 - dashed lines - rarefaction waves 


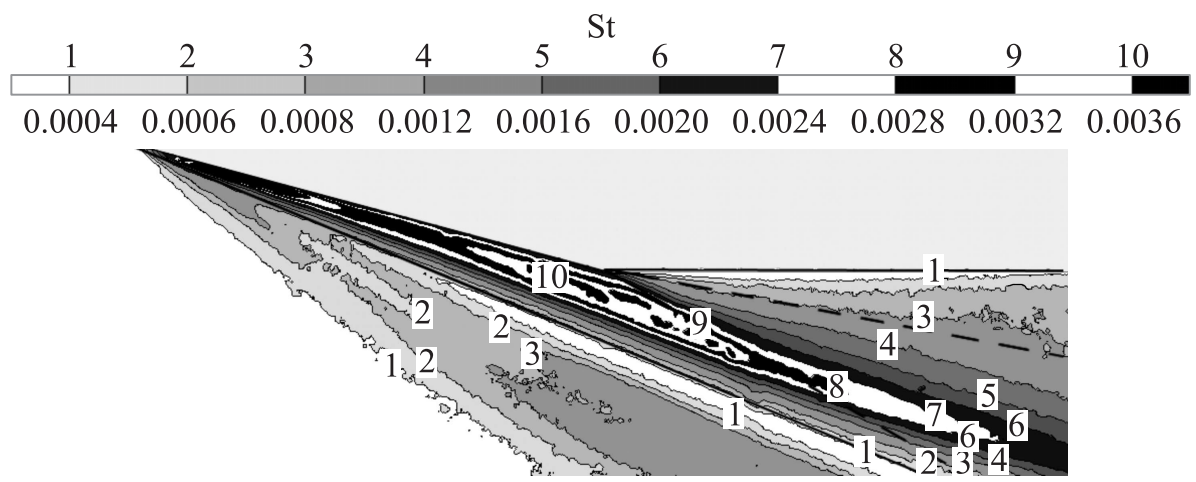

(a)

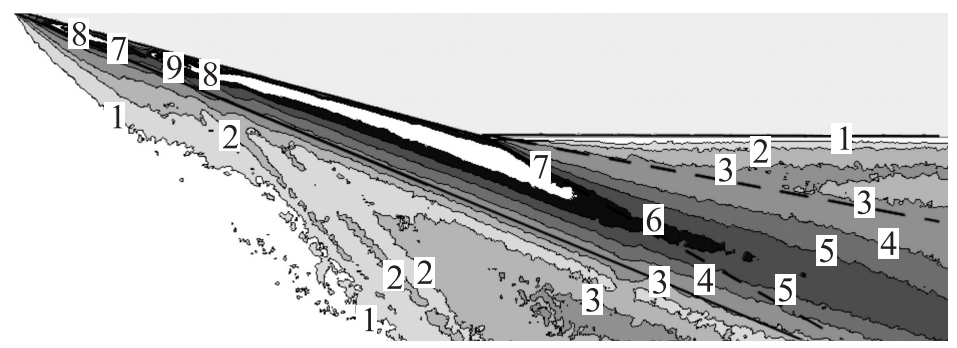

(b)

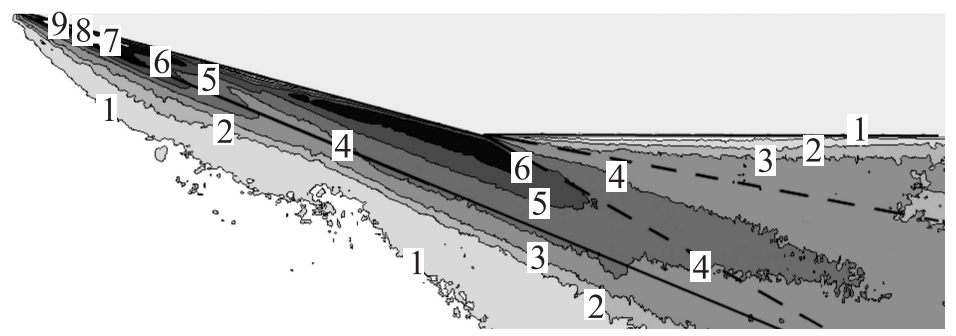

(c)

Figure 4 Stanton number isolines on the plate in the neighborhood of wedge with $\theta=15^{\circ}$ at $\operatorname{Re}_{\infty, L}=4.7 \cdot 10^{6}:(a) r=0$; (b) 0.5 ; and (c) $r=1 \mathrm{~mm}$

pressure, friction, and heat transfer increase sharply in the reattachment region (in Figs. 3 and 4, the solid line corresponds to the shock wave while the dashed lines correspond to the rarefaction waves). The pressure increase starts at a significant distance ahead of the shock.

Analyzing the pressure distribution in Fig. 3, one must point out that pressure increase does not take place ahead of the shock near the wedge's leading edge 
(see isobars in Figs. $3 a$ and $3 b$ ) but starts only at some distance from the leading edge, and the blunting of the plate moves this zone downstream.

The method used for surface flow visualization provides reliable information only in the regions of high friction. The line of primary separation taking place far ahead of the shock is not visible. The reattachment line (the divergent line 1) and the line of secondary separation which practically coincides with the shock are clearly visible in Fig. $3 c$. It is notable that the divergent line is also formed only at some distance behind the wedge's leading edge. The blunting of the plate additionally moves the position of this line downstream. Thus, the pressure measurements and surface flow visualization reveal that blunting of the plate causes broadening of the nonconical region near the wedge's leading edge.

Distributions of Stanton numbers near the wedge $\theta=15^{\circ}$ (see Fig. 4) on the plates with different bluntness are characterized by certain features of heat transfer in the interference region. A narrow zone of enhanced heat transfer is formed between the shock and the wedge. Another narrow zone of local heat transfer weakening arises near the secondary-separation line (i. e., near the shock wave shown as solid line in Fig. 4). The zone of enhanced heat transfer extends far behind the wedge's corner. Between the first and the last rarefaction lines originating from the corner point, the heat flux diminishes slowly and approaches the undisturbed value only at the wedge's surface parallel to the undisturbed flow direction.

Even a small bluntness of the plate results in significant weakening of heat flux in the interference region. The zone of enhanced heat transfer diminishes and the maximum Stanton number decreases at the radius $r$ increase. The zones of extremely high heat transfer (typical of the sharp plate) disappear at $r \geq 1 \mathrm{~mm}$ (Fig. 4c).

The obtained data demonstrate one more feature of the investigated flow. A periodic system of strips (waves) with enhanced and weakened heat transfer is formed on the plates with bluntness radius from 0.1 to $0.5 \mathrm{~mm}$ at $\operatorname{Re}_{\infty, L}=4.7$ $\times 10^{6}$. It is located in the outer part of the interference region, between the lines of primary and secondary separation. Such strips are visible in Fig. $4 b$ which shows Stanton number distribution at $r=0.5 \mathrm{~mm}$. Up to 4 strips can be distinguished at some radius values. The maximum value of Stanton number in the middle of a strip exceeds the minimum value between the strips by $25 \%-60 \%$. The distance between the strips (the wave length) in the undisturbed flow direction is $5-6 \mathrm{~mm}$ which is significantly larger than the boundary layer thickness $(\delta \sim 1 \mathrm{~mm})$. The inclination angle of the strips relative to the undisturbed flow direction is approximately $50^{\circ}$. It means that the strips are approximately parallel to the line along which starts the increase of heat transfer. Possibly, the formation of the strips is caused by the nonstability of the boundary layer in the preseparation zone ahead of the shock.

Figure 5 demonstrates the influence of bluntness of the plate on heat flux distribution. Intensification of heat transfer starts at $\varphi \approx 50^{\circ}$ (see Fig. 1), 
i. e., far ahead of the shock, outside the zone shown in Fig. 5. At $r=0$, a plateau with the approximately constant value of heat transfer coefficient is formed immediately ahead of the shock (the vertical dashed line corresponds to the shock). Stanton number achieves its maximum value near the wedge's surface, on the ray $\varphi-\theta$ $=1.5^{\circ}-3^{\circ}$ situated in the vicinity of the diverging line. The maximum Stanton number value $\mathrm{St}_{m}$ exceeds the plateau value 4-6 times at $\operatorname{Re}_{\infty, L}=4.7 \cdot 10^{6}$ and $2.5^{-}$ 3 times at $\operatorname{Re}_{\infty, L}=19.2 \cdot 10^{6}$. The minimum St is formed in the vicinity of the secondary separation line. This minimum on a sharp plate is stronger than on blunted plates (lines 2-4).

Blunting of the plate leads to a significant decrease of $\mathrm{St}_{m}$. Simultaneously, the zone of local decrease of Stanton number is moved from the wedge, i.e., the zone of enhanced heat transfer is widened. The widening of this zone becomes significant at a larger bluntness radius $r \geq 2$.

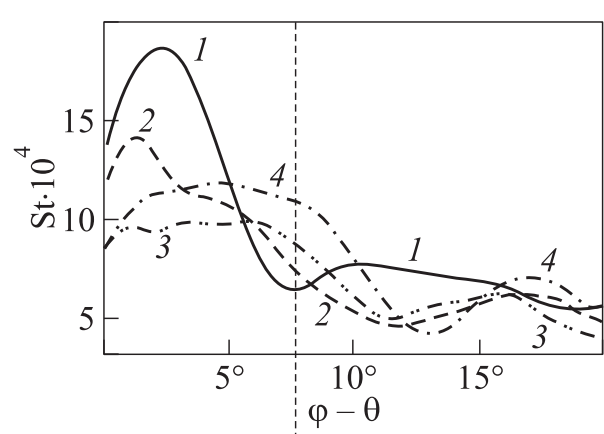

(a)

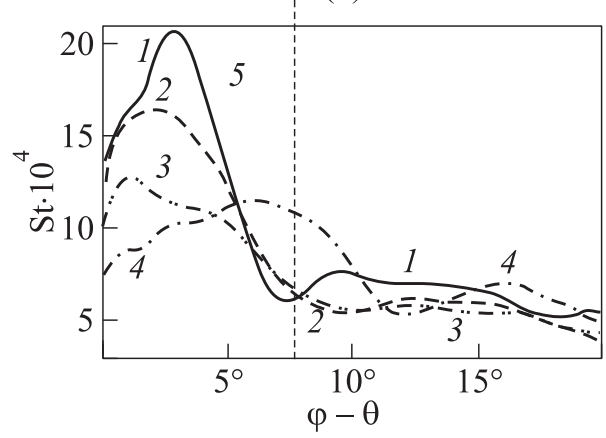

(b)

Figure 5 Transversal distribution of Stanton number on sharp and blunt plates near the wedge with $\theta=15^{\circ}$ at $\operatorname{Re}_{\infty, L}=19.2 \cdot 10^{6}$ : (a) $R / X_{0}=0.46$, (b) $R / X_{0}=0.69 ; 1-r$ $=0 \mathrm{~mm} ; 2-0.3 ; 3-0.75$; and $4-r$ $=2 \mathrm{~mm}$

\subsection{Influence of Shock Strength and Bluntness of the Plate on the Maximum Amplification of Heat Transfer in the Interference Region}

The maximal value of Stanton number in the cross section $R / X_{0}=0.46$ normalized on the value $\mathrm{St}_{t}$ calculated for the turbulent boundary layer at point $X=X_{0}$ is shown in Fig. 6. It is presented vs. the ratio of the pressure $P_{2}$ behind the oblique shock to the pressure $P_{1}$ ahead it for the inviscid flow $\left(\xi=P_{2} / P_{1}\right)$. The data are approximated as [8]:

$$
\frac{\mathrm{St}_{m}}{\mathrm{St}_{\mathrm{ot}}}=A \xi^{n} .
$$




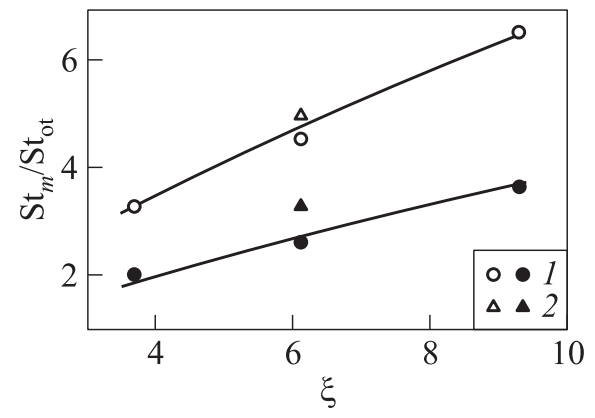

Figure 6 Influence of shock strength on maximal heat transfer augmentation in the cross section $R / X_{0}=0.46$ (empty signs refer to $r=0 \mathrm{~mm}$ and black signs to $r=1 \mathrm{~mm}): 1-\operatorname{Re}_{\infty, L}=19.2 \cdot 10^{6}$; and $2-\operatorname{Re}_{\infty, L}=4.7 \cdot 10^{6}$

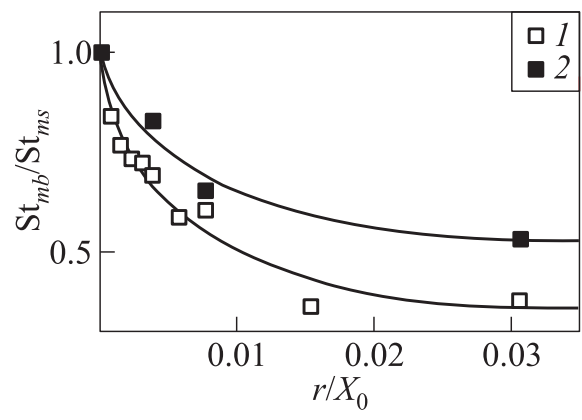

Figure 7 The influence of bluntness on the maximum Stanton number in the cross section $R / X_{0}=0.69: 1-\operatorname{Re}_{\infty, L}=$ $4.7 \cdot 10^{6}$, and $2-\operatorname{Re}_{\infty, L}=19.2 \cdot 10^{6}$

For the sharp and blunted plates, the value $n=0.74$ is obtained. At that, for the sharp plate, $A=1.24$, and for the blunted one $(r=1 \mathrm{~mm}), A=0.71$. In [8], for the sharp plate at $\mathrm{M}=5$ and $\operatorname{Re}_{\infty, L}=18.5 \cdot 10^{6}$, the values $n=0.8992$ and $A=1.1086$ are obtained. It is evident that blunting of the plate leads to a significant decrease of $\mathrm{St}_{m}$.

At lower Reynolds number $\operatorname{Re}_{\infty, L}=4.7 \cdot 10^{6}$, the data are obtained only for the wedge with the angle $\theta=15^{\circ}(\xi=6.1)$. In this case, the undisturbed boundary layer is laminar and the ratio $\mathrm{St}_{m} / \mathrm{St}_{t}$ is much larger (approximately 3 times) than at $\operatorname{Re}_{\infty, L}=19.2 \cdot 10^{6}$. But if the value $\mathrm{St}_{m}$ measured at the laminar flow (at $\operatorname{Re}_{\infty, L}=4.7 \cdot 10^{6}$ ) is normalized on the value $\mathrm{St}_{\text {ot }}$ calculated at point $X=X_{0}$ for the turbulent boundary layer, the ratio $\mathrm{St}_{m} / \mathrm{St}_{\text {ot }}$ at $\mathrm{Re}_{\infty, L}=4.7 \cdot 10^{6}$ is $12 \%-38 \%$ higher than at $\operatorname{Re}_{\infty, L}=19.2 \cdot 10^{6}$ (see Fig. 6). Thus, the following conclusions can be made: (i) at lower Reynolds number $\operatorname{Re}_{\infty, L}=4.7 \cdot 10^{6}$, the laminar-turbulent transition happens inside the interference region; and $(i i)$ in this case, the maximum value of the heat transfer coefficient reaches a higher value than at the transition ahead of the interference region.

The dependence of $\mathrm{St}_{m}$ on the radius of bluntness $r$ is presented in Fig. 7 for the wedge with $\theta=15^{\circ}$. Here, the maximum value $\mathrm{St}_{m b}$ on the blunted plate is related to a similar value on the sharp plate $\mathrm{St}_{m s}$ at the same flow conditions, and the bluntness radius is related to the distance $X_{0}$ from the plate leading edge to the wedge's leading edge. Similarly to $2 \mathrm{D}$ flow, even small blunting causes significant decrease of $\mathrm{St}_{m}$ value. This decrease takes place only up to some value of normalized bluntness $r / X_{0}=0.02-0.03$ which can be designated as threshold bluntness, by analogy with 2D flow [11]. It must be emphasized that in 
the described experiments, the wedge's leading edge is placed far from the plate leading edge and the circular cross section under consideration $X / X_{0}=0.69$ is placed even farther.

Figure 7 also demonstrates the weakening of the influence of the high entropy layer on heat transfer in the interference region at Reynolds number $\operatorname{Re}_{\infty, L}=19.2 \cdot 10^{6}$ in comparison with $\operatorname{Re}_{\infty, L}=4.7 \cdot 10^{6}$ at all tested bluntness radii including high values of $r$. Apparently, the difference is related to the peculiarities of the interaction between the laminar and turbulent boundary layers with the high entropy layer. This problem deserves the further study.

\section{CONCLUDING REMARKS}

When the boundary layer ahead of the wedge is laminar, the maximum value of the Stanton number behind the shock reaches approximately the same level as in the case of the turbulent boundary layer or even exceeds it.

Small bluntness of the plate results in significant decrease of the maximum Stanton number in the interference zone and in some widening of the high heating region.

The decrease of heat transfer in the interference zone due to the bluntness of the plate takes place only in the narrow range of the bluntness radius (at $\left.r / X_{0}<0.02-0.03\right)$. At $r / X_{0}>0.03$, the influence of the bluntness on heat transfer is negligible.

The panoramic methods based on the use of luminescent paints are effective in investigations of heat transfer and pressure distribution in short duration wind tunnels.

\section{ACKNOWLEDGMENTS}

The work was performed with the support of the Russian Foundation for Basic Research (Project No. 08-01-00449).

\section{REFERENCES}

1. Zheltovodov, A. A. 2004. Advances and problems in modeling of shock wave turbulent boundary layer interactions. 12th Conference (International) Methods of Aerophysics Research. Novosibirsk. 2:225-36.

2. Borovoy, V. Ya., and E. V. Sevast'yanova. 1973. Gas flow and heat transfer in the zone of interaction between a laminar boundary layer and a shock wave in the neighborhood of a half-wing mounted on a plate. Uchen. Zapiski TsAGI 4(2):5463. [In Russian.]

3. Zheltovodov, A. A. 1979. Properties of two- and three-dimensional separation flows at supersonic velocities. Fluid Dyn. 14(3):357-64. 
4. Borovoy, V. Ya. 1983. Gas flow and heat transfer in zones of interaction between shock waves and boundary layer. Moscow: Mashinostroenie. 142 p. [In Russian.]

5. Kussoy, M.I., K. C. Horstman, and C. C. Horstman. 1993. Hypersonic crossing shock-wave/turbulent-boundary-layer interactions. AIAA J. 31(12):2197-203.

6. Garrison, T. J., G. S. Settles, N. Narayanswam, and D. D. Knight. 1994. Laser interferometer skin-friction measurements of crossing-shock-wave/turbulent-boundarylayer interactions. AIAA J. 32(6):1234-41.

7. Knight, D., H. Yan, A. G. Panaras, and A. Zheltovodov. 2003. Advances in CFD prediction of shock wave turbulent boundary layer interactions. Progr. Aerospace Sci. 39(2-3):121-84.

8. Schulein, E. 2006. Skin-friction and heat flux measurements in shock/boundarylayer interaction flows. AIAA J. 44(8):1732-41.

9. Borovoy, V. Ya., I. V. Egorov, A. S. Skuratov, and I. V. Struminskaya. 2005. Effect of a high-entropy layer on heat transfer in the region of the incidence of an oblique shock wave on a blunted-plate surface. Doklady Phys. 50(1):3-6.

10. Borovoy, V. Ya., I. V. Egorov, A.S. Skuratov, and I. V. Struminskaya. 2005. Interaction between an inclined shock and boundary and high entropy layers on a flat plate. Fluid Dyn. 40(6):911-28.

11. Borovoy, V. Ya., A. S. Skuratov, and I. V. Struminskaya. 2008. On the existence of a threshold value of the plate blunting in the interference of an oblique shock with boundary and high entropy layers. Fluid Dyn. 43(3):369-79.

12. Borovoy, V., V. Mosharov, A. Noev, and V. Radchenko. 2009. Laminar-turbulent flow around a wedge placed on sharp and blunted plates. Fluid Dyn. 44(3):58-74.

13. Merski, N.R. 1998. Reduction and analysis of phosphor thermography data with the IHEAT software package. AIAA Paper No. 98-0712.

14. Borovoy, V., V. Mosharov, V. Radchenko, and A. Noev. 2007. Three-dimensional interference of oblique shock with boundary and high-entropy layers and technique intended for investigation of such flows. West-East High Speed Flow Field Conference. Moscow, Russia.

15. Borovoy, V., V. Mosharov, A. Noev, and V. Radchenko. 2009. Temperature Sensitive Paint application for investigation of boundary layer transition in shortduration wind tunnels. EUCASS.

16. Kautsky, H., and A. Hirsch. 1935. Nachweis geringster Sauerstoffmengen durch Phosphoreszenztilgung. Z. anorg. Chem. 222(2):126-34.

17. Ardasheva, M. M., L. B. Nevsky, and G. E. Pervushin. 1985. Method of measurement pressure distribution by the help of pressure sensitive coating. PMTF 4:24-30. [In Russian.]

18. Borovoy, V., A. Bykov, V. Mosharov, A. Orlov, V. Radchenko, and S. Fonov. 1995. Pressure sensitive paint application in shock wind tunnel. 16th ICIASF Congress Dayton, Ohio.

19. Mosharov, V.E., V.N. Radchenko, and A.A. Orlov. 2006. Method of visualization gas and fluid flows over an objects surface. Patent of RF №2288476. Priority 14.03.2005. Published 27.11.2006.

20. Mosharov, V.E., A. A. Orlov, and V.N. Radchenko. 1985. Application of correlation analysis in surface flow visualization with oil film. SPIE Proceedings. Bellingham, WA. 62620B. 